\title{
Is your grommet really necessary?
}

Otitis media with effusion or glue ear is one of the most common chronic conditions affecting children world wide. Diagnosis is difficult in young children. Diagnostic techniques should include use of pneumatic otoscopy, tympanometry, and pure tone audiometric or other hearing assessments, according to age. The incidence and prevalence of the conditions vary, depending on the technique used. The prevalence, however, decreases as age increases, being high in 1-2 year olds and low in children over the age of 10; the peak seems to be in children aged 1 to 5 years.

The condition can be mild, moderate, or severe. Most mild and many moderate cases resolve spontaneously within weeks or months. Our investigations suggest that up to one third of moderate to severe cases may also resolve if follow up is continued for more than three months. Many, however, run a relapsing and remitting course, which may last for several years.

At present there seems to be no medical treatment that will effect a long term cure. Various operations have been advocated, both to produce a cure and to prevent recurrence. Adenoidectomy alone or in combination with tonsillectomy and insertion of a grommet or ventilation tube are the most frequently advised, though there seems little evidence to support the addition of tonsillectomy with removal of the adenoids. ${ }^{1}$ The number of operations being performed for glue ear both in this country and abroad has increased in recent years. ${ }^{2}$ At present there is no satisfactory definition of the condition to allow comparative studies of methods of treatment to be made between centres. Most serious investigators, however, require a minimum period of observation of three months, with more than one examination by a qualified pneumatic otoscopist. Tympanometry and pure tone audiometric studies should be used when possible for diagnosis and for assessment during follow up.

Mild, transient forms of the condition with slight hearing loss, especially if unilateral, almost certainly do not require treatment. Moderate forms, especially if bilateral, may affect speech and language development in younger children. Schooling and behaviour are also affected in older children, and if effusions persist for more than three months in association with symptoms intervention is probably required. In such cases the seasonal variation should be considered when treatment is planned. Severe forms of the condition, especially if bilateral, will invariably require treatment. In such cases the mean audiometric hearing thresholds are usually in excess of 30 decibels in each ear. Naturally the state of the tympanic membrane in terms of retraction, scarring, and atelectasis requires consideration.

Most studies reporting treatment by adenoidectomy, adenotonsillectomy, or insertion of grommets have been of unsatisfactory design and have failed to evaluate each procedure in isolation. Often the effects of adenoidectomy and insertion of grommets have not been analysed separately, neither has a control group treated without operation been included. Many studies have been of short duration and have not taken account of seasonal effect on outcome. Our investigations have tried to overcome these defects in study design, and we have sufficient data, including those obtained during follow up for at least three years, for satisfactory postoperative evaluation. Information obtained during follow up for five years is accumulating for sufficient numbers of patients to indicate certain trends. We investigated clearance of effusions by pneumatic otoscopy, changes in results of impedance studies, and hearing gain on pure tone audiometry. After one year there was no significant difference between any of the treatments in isolation, and this effect persisted for at least three years. In the group that received no treatment there was a slow spontaneous resolution of the effusion with a concurrent change in impedance and hearing gain. At three years fluid was still present in $40 \%$ of ears and there was a mean hearing loss of 22 decibels, compared with a preoperative threshold of 31 decibels. The addition of tonsillectomy to adenoidectomy seemed not to confer any extra benefit, and there was no significant difference between adenoidectomy alone and insertion of a grommet. To achieve the same effect with a grommet alone, however, reinsertion was required twice as often as when the procedure was combined with adenoidectomy. ${ }^{3}$

\section{Insertion of grommets}

Insertion of a grommet has two clinical indications. Firstly, it may be a short term measure to ventilate the middle ear when there are reversible changes in the middle ear and tympanic membrane. In these cases there is temporary dysfunction of the eustachian tube; this is the case in almost all children 
suffering from glue ear. Ultimately the child outgrows this underlying dysfunction and the condition resolves. A grommet may, however, need to be reinserted in $30-50 \%$ of cases, and in some cases revision is required on three or more occasions. The second clinical group have permanent dysfunction of the eustachian tube and irreversible changes of the tympanic membrane, often showing degrees of collapse or atelectasis. In these cases complete resolution is impossible and longer term ventilation is required. The rate of complications in this group both with and without treatment is high. At present there is no method of detecting, either in infancy or at the outset of the condition, which children will fall into this clinical group.

\section{Short term ventilation}

Advantages. Short term ventilation results in immediate improvement in hearing and fairly rapid correction of mucosal changes in the middle ear. Any subjective pressure effect or feeling of blockage resolves. Tinnitus often disappears, and sometimes dizziness, if present, will be controlled. Behavioural problems often improve, with later obvious improvement in speech, language, and learning. The effects last for six to 12 months. The procedure requires general anaesthesia but not intubation, so the child can be admitted, have the operation and be discharged the same day. There is clearly some cost benefit compared with other surgical procedures that require admission overnight. There may also be additional benefit for those children prone to recurrent acute exacerbations of suppurative otitis media as well as the underlying middle ear effusion. In these the frequency of otitis may be reduced while the grommet remains patent. ${ }^{4}$

Disadvantages. The patency of a grommet and the period of retention in the tympanic membrane are insufficient to correct the natural history of the disease in at least $30 \%$ of children. This is particularly so in younger children, in whom spontaneous resolution may not be expected for several years and the reinsertion rate may be much higher than that in older children. At the outset of treatment it is advisable to inform the parents of the probable need for reinsertion, which will require further anaesthesia.

There are immediate complications from inserting a grommet. A few patients develop otorrhoea, which is usually transitory but in some cases is longstanding and requires treatment. A few children develop otalgia. Damage to middle ear structures and even to the inner ear has been reported, either as a result of myringotomy and insertion of the grommet or from the agents used to clean the meatus before surgery. In some children secondary otitis media may develop after swimming, but this is rare. It is advisable, however, to recommend that children with grommets may continue to swim but should avoid diving or jumping into the water. Soapy bath water may be more troublesome than swimming pool water.

Later complications comprise scarring and loss or damage of the middle layer of the tympanic membrane at the site of the grommet. Thin triangular segmental defects are often visible after extrusion. Tympanosclerosis to a variable degree may be seen in as many as $40 \%$ of cases after one year and in $70 \%$ after two years. ${ }^{5}$ It is rarely seen in an ear that has not been operated on, and is much less common when myringotomy alone is performed. Tympanosclerosis tends to worsen with time, and our studies suggest that after five years there is a slight but detrimental effect on hearing ability in those patients with the most severe forms of sclerosis when compared with that of the contralateral ear that has not been operated on. This finding is supported by the longer term follow up studies of Gundersen et al. ${ }^{6}$ In a few cases persistent perforation of the tympanic membrane results. There seems little doubt from the studies by Tos et al that insertion of a grommet fails to resolve development of attic retraction in cases of glue ear. ${ }^{7}$ This suggests that short term ventilation lacks any protective effect on the possibility of cholesteatoma developing in the long term.

\section{Long term ventilation}

The question of longer term ventilation with specially designed tubes in cases of irreversible dysfunction of the eustachian tube and changes in the middle ear requires further study.

\section{Summary}

There is little doubt that surgical treatment is required for many moderate to severe cases of glue ear, particularly if the condition is bilateral, present for more than three months, and associated with considerable subjective hearing loss. Present evidence suggests that the choice rests between insertion of a grommet or ventilation tube and adenoidectomy. We have shown resolution of $40 \%$ of effusions assessed one year after removal of the adenoids, and our data at three years show further improvement. $^{3}$ There is also evidence that adenoidectomy in cases selected by age and postnasal airway size may produce resolution in as many as $70 \%{ }^{8}$ This single procedure is associated with 
substantial morbidity and mortality and requires an overnight stay in hospital. By contrast, a grommet can be inserted on a day case basis and is accompanied by less risk. If it is used in isolation, however, readmission to hospital for reinsertion will be required in a high proportion of cases. The immediate gain in hearing and subjective benefit from insertion of a grommet is obvious to all clinicians responsible for such children. By contrast, the hearing gain after adenoidectomy is delayed for some weeks. The tympanic membrane changes caused by a grommet may, however, result in some cases in a slight but permanent hearing loss, which does not occur after adenoidectomy.

A compromise regimen for treatment in bilateral cases may be adenoidectomy, after consideration of age and size of postnasal airway, combined with unilateral insertion of a grommet. In this way the longer term benefit of the single procedure of adenoidectomy is combined with the shorter term benefit of hearing gain that results from immediate reventilation of the middle ear. The use of only one grommet reduces the overall complication rate by half. Naturally myringotomy and aspiration of the contralateral effusion without insertion of a grommet would be carried out under the same anaesthetic.

\section{References}

1 Maw AR, Herod F. Otoscopic, impedance and audiometric findings in glue ear treated by adenoidectomy and tonsillectomy. Lancet 1986;i:1399-402.

2 Black N. Surgery for gluc ear - a modern epidemic. Lancet 1984;ii:835-7.

3 Maw AR. Tonsils and adenoids, their relation to secretory otitis media. In: Cremers C. ed. Recent advances in Otorhinolaryngology. Basle:Karger (in press).

+ Gonzalez C. Washington DC, Arnold JE, et al. Prevention of recurrent acute otitis media: chemophrophylaxis versus tympanostomy tubes. Laryngoscope 1986;96:1330-4.

${ }^{5}$ Slack RWT, Maw AR, Capper JWR, Kelly S. Prospective study of tympanosclerosis developing after grommet insertion. $J$ Laryngol Otol 1984:98:771-4.

${ }^{\circ}$ Gundersen T, Tonning FM, Kveberg KH. Ventilating tubes in the middle ear. Long term observations. Arch Otolaryngol 1984:110:783-4.

7 Tos M. Stangerup SE. Holm-Jensen S. Sorensen CH. Spontaneous course of secretory otitis and changes of the ear drum Arch Otolaryngol 1984:110:281-9.

* Maw AR. Adenoidectomy and adenotonsillectomy for otitis media with effusion (glue ear) in children. A prospective randomized controlled study. London: University of London, 1986. (MS thesis) Pp 157.

A R MaW

Consultant Otolaryngologist, Royal Hospital for Sick Children and Bristol Royal Infirmary, Bristol. 